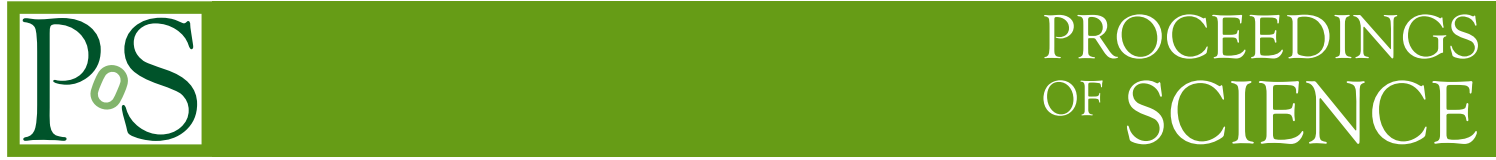

\title{
Combination of CDF and DO measurements of the W boson helicity in top quark decays
}

\author{
E.W. Varnes* \\ University of Arizona \\ E-mail: varnes@physics.arizona.edu
}

I report on the combination of measurements from CDF and D0 of the helicity fractions of $W$ bosons from top quark decays. The result, when both the longitudinal $\left(f_{0}\right)$ and right-handed $\left(f_{+}\right)$fractions are allowed to float, is $f_{0}=0.722 \pm 0.081[ \pm 0.062$ (stat.) \pm 0.052 (syst.)] and $f_{+}=-0.033 \pm 0.046[ \pm 0.034$ (stat.) \pm 0.031 (syst.) $]$, consistent with the Standard Model (SM) expectation. When one fraction is fixed to its SM value the results for the other fraction are $f_{0}=0.682 \pm 0.057[ \pm 0.035$ (stat.) \pm 0.046 (syst.) $]$ and $f_{+}=-0.015 \pm 0.035[ \pm 0.018$ (stat.) \pm 0.030 (syst.)].

36th International Conference on High Energy Physics,

July 4-11, 2012

Melbourne, Australia

\footnotetext{
*Speaker.
} 


\section{Introduction}

One of the highlights of Run II of the Tevatron was the continual improvement in measurements of the properties of the top quark. Such measurements are of interest because the top is the most recently discovered of the quarks [1], and because its large mass indicates that it is the only fermion with a coupling strength of $\mathscr{O}(1)$ to the Higgs field. Among the properties measured by both the CDF and D0 experiments are the helicity fractions of the $W$ bosons produced in top quark decays. In the standard model (SM) these are expected to be $f_{0}=0.688 \pm 0.004$, $f_{-}=0.310 \pm 0.004$, and $f_{+}=0.0017 \pm 0.0001$ [2] where $f_{0}, f_{-}$, and $f_{+}$are the longitudinal, lefthanded, and right-handed fractions, respectively. Any deviation from these values would indicate the presence of new physics. Since measurements of the fractions at the Tevatron are statistically limited, the precision can be improved substantially by combining the results from CDF and D0 [3].

\section{Input Measurements}

The combination takes as input two measurements from CDF, one using $t \bar{t}$ events in the lepton plus jets channel [4] and the other using events in the dilepton channel [5], and a measurement from D0 that uses both lepton plus jets and dilepton events [6]. These measurements are summarized in Table 1 and come in two forms: "two-dimensional" (2D) measurements in which both $f_{0}$ and $f_{+}$are allowed to vary simultaneously, and "one-dimensional" (1D) measurements in which one of these parameters is fixed to its SM value while the other is varied. CDF and D0 adopted different procedures for treating the uncertainty in the top quark mass $m_{t}$. For the purpose of the combination CDF reinterpreted their results using D0's procedure, so the values in Table 1 differ slightly from those in [4] and [5]. The statistical uncertainty accounts for a large fraction of the total uncertainty in each measurement, which motivates the combination of the results to achieve the best possible precision even with the presence of correlated systematic uncertainties.

\section{Systematic Uncertainties}

The combination requires proper accounting of the systematic uncertainties and their correlations. To facilitate this, the uncertainties are separated into categories such that there may be correlations between the uncertainties within one category but not between different categories. The simplest are uncertainties that are uncorrelated between any two analyses (MTD), and the uncertainties from limited MC statistics and from analysis closure tests fall into this category. Next are uncertainties that are correlated between analyses within an experiment but uncorrelated between experiments (DET). Uncertainties in jet reconstruction efficiency and energy resolution are in this category, as are muon efficiency uncertainties specific to D0. In principle uncertainties related to the modeling of multiple $p \bar{p}$ interactions in a crossing (MHI) would fall into this category, but this effect is treated as a systematic uncertainty only in CDF's dilepton analysis, while in the other analyses these effects are included in the nominal Monte Carlo model. The rest of the systematics are treated as fully correlated between analyses and experiments. The first such uncertainty (JES) arises from the jet energy calibration [7,8], including uncertainties specific to the calibration of $b$ jets (while jet energy calibration includes components that are uncorrelated between experiments, 
it is assumed here that the theoretical uncertainties in describing jets are dominant. The results of the combination would change negligibly if a different assumption was made). The next is the uncertainty on the measured top quark mass [9](MTOP). Several effects that impact the modeling of $t \bar{t}$ events (SIG) are also considered, including uncertainties parton distribution functions, gluon radiations, $b$ quark fragmentation, and parton shower modeling. Uncertainties in background modeling (BGD), including uncertainties in the fraction of heavy flavor jets in $W+$ jets production, are considered as well.

Where correlations exists, they are assumed to be have maximal magnitude. The correlation coefficient between any two correlated measurements of a given helicity fraction is taken to be 1 , while the correlation coefficient between correlated measurements of different helicity fraction is taken to be -1 , reflecting the large statistical anti-correlation between $f_{0}$ and $f_{+}$.

\section{Combination Procedure}

The measurements are combined using the best linear unbiased estimator approach $[10,11]$. This method takes into account all sources of uncertainty by forming the complete covariance matrix for each measurement, obtained from the sum of the covariance matrices corresponding to each category of uncertainty.

Table 1: Summary of the $W$ boson helicity measurements used in the combination of results. The CDF measurements have been shifted from their published values to reflect a change in the assumed top quark mass from 175 to $172.5 \mathrm{GeV} / \mathrm{c}^{2}$. The first uncertainty in brackets below is statistical and the second is systematic.

\begin{tabular}{cc}
\hline \hline CDF lepton + jets, $2.7 \mathrm{fb}^{-1}[4]$ & \\
$f_{0}^{2 \mathrm{D}}$ & $f_{0}=0.903 \pm 0.123[ \pm 0.106 \pm 0.063]$ \\
$f_{+}^{2 \mathrm{D}}$ & $f_{+}=-0.195 \pm 0.090[ \pm 0.067 \pm 0.060]$ \\
\hline$f_{0}^{1 \mathrm{D}}$ & $f_{0}=0.674 \pm 0.081[ \pm 0.069 \pm 0.042]$ \\
$f_{+}^{1 \mathrm{D}}$ & $f_{+}=-0.044 \pm 0.053[ \pm 0.019 \pm 0.050]$ \\
\hline CDF dilepton, 5.1 fb ${ }^{-1}[5]$ & \\
$f_{0}^{2 \mathrm{D}}$ & $f_{0}=0.702 \pm 0.186[ \pm 0.175 \pm 0.062]$ \\
$f_{+}^{2 \mathrm{D}}$ & $f_{+}=-0.085 \pm 0.096[ \pm 0.089 \pm 0.035]$ \\
\hline$f_{0}^{1 \mathrm{D}}$ & $f_{0}=0.556 \pm 0.106[ \pm 0.088 \pm 0.060]$ \\
$f_{+}^{1 \mathrm{D}}$ & $f_{+}=-0.089 \pm 0.052[ \pm 0.041 \pm 0.032]$ \\
\hline D0, lepton + jets and & \\
dilepton, 5.4 fb ${ }^{-1}[6]$ & \\
$f_{0}^{2 \mathrm{D}}$ & $f_{0}=0.669 \pm 0.102[ \pm 0.078 \pm 0.065]$ \\
$f_{+}^{2 \mathrm{D}}$ & $f_{+}=0.023 \pm 0.053[ \pm 0.041 \pm 0.034]$ \\
\hline$f_{0}^{1 \mathrm{D}}$ & $f_{0}=0.708 \pm 0.065[ \pm 0.044 \pm 0.048]$ \\
$f_{+}^{1 \mathrm{D}}$ & $f_{+}=0.010 \pm 0.037[ \pm 0.022 \pm 0.030]$ \\
\hline \hline
\end{tabular}




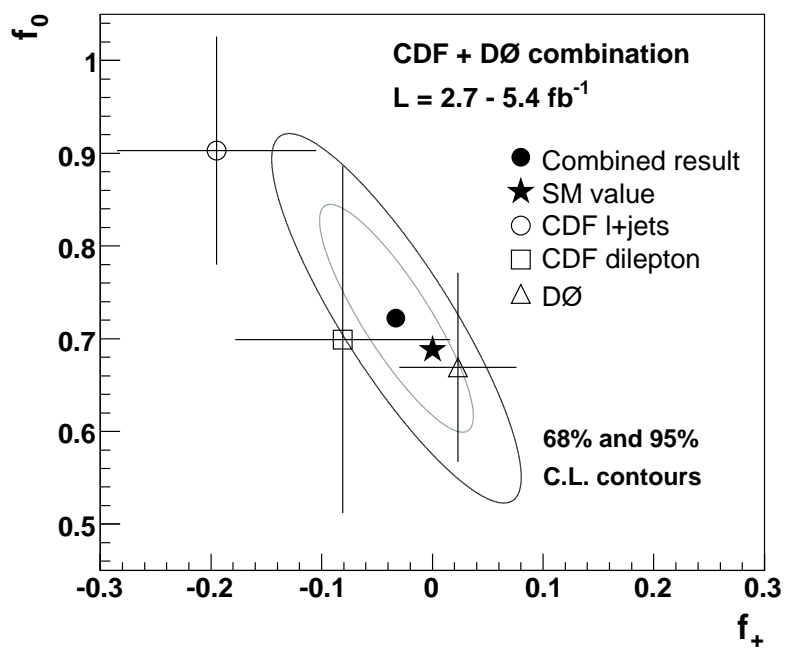

Figure 1: Contours of constant $\chi^{2}$ for the combination of the 2D helicity measurements. The ellipses indicate the $68 \%$ and $95 \%$ C.L. contours, the dot shows the best-fit value, and the star marks the expectation from the SM. The input measurements to the combination are represented by the open circle, square, and triangle, with error bars indicating the $1 \sigma$ uncertainties on $f_{0}$ and $f_{+}$. Each of the input measurements uses a central value of $m_{t}=172.5 \mathrm{GeV} / c^{2}$.

\section{Results}

The combination of the $2 \mathrm{D}$ fits gives $f_{0}=0.722 \pm 0.081[ \pm 0.062$ (stat.) \pm 0.052 (syst.) $], f_{+}=$ $-0.033 \pm 0.046\left[ \pm 0.034\right.$ (stat.) \pm 0.031 (syst.)], as shown in Fig. 1 . The $\chi^{2}$ of the combination is 8.86 for four degrees of freedom, meaning that the measurements are consistent with each other within two standard deviations (s.d.). The correlation coefficient between the combined values of $f_{0}$ and $f_{+}$is -0.86 . The combination of $1 \mathrm{D}$ measurements with $f_{0}$ fixed gives $f_{+}=-0.015 \pm 0.035[ \pm 0.018$ (stat.) \pm 0.030 (syst.) $]$, and the combination with $f_{+}$fixed gives $f_{0}=0.682 \pm 0.057[ \pm 0.035$ (stat.) \pm 0.046 (syst.) $]$, as shown in Fig. 2 .. The $\chi^{2}$ for consistency among the input $f_{0}\left(f_{+}\right)$measurement is 2.12 (4.44) for two degrees of freedom. The contribution of each source of uncertainty to the combined result is shown in Table 2. The results of both the 2D and 1D combinations are consistent with the SM within one s.d.

\section{Summary and Outlook}

CDF and D0 have combined their measurements of the $W$ boson helicity fractions in top quark decays. The combinations shows that there is reasonable consistency between CDF and D0's measurements, and that the combined values are consistent with SM expectations. This is the first combination of top quark results carried out by the CDF and D0 collaborations themselves. The measurements reported here make use of about half of the Tevatron dataset; both the CDF and D0 experiments are preparing updated results using the full $\approx 10 \mathrm{fb}^{-1}$ Run II sample. 

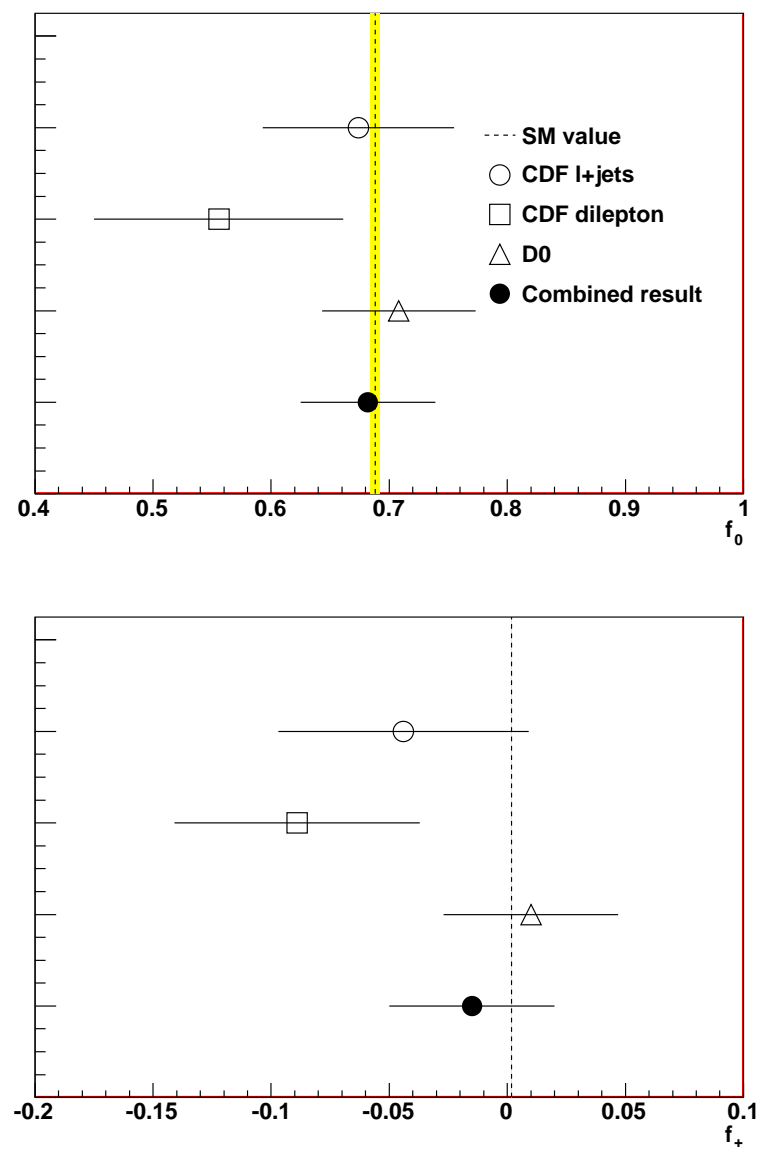

Figure 2: Summary of the individual 1D measurements and the combined result for $f+0$ (upper plot) and $f_{+}$(lower plot). The dashed line is at the SM value for each fraction, with the yellow band representing the uncertainty on the SM value.

Table 2: The contribution from each category of systematic uncertainty in the combined measurements.

\begin{tabular}{lcccc}
\hline \hline Category & \multicolumn{2}{c}{ 2D combination } & \multicolumn{2}{c}{ 1D combination } \\
& $\delta f_{0}$ & $\delta f_{+}$ & $\delta f_{0}$ & $\delta f_{+}$ \\
\hline JES & 0.007 & 0.012 & 0.018 & 0.014 \\
SIG & 0.038 & 0.022 & 0.036 & 0.021 \\
BGD & 0.028 & 0.013 & 0.012 & 0.009 \\
MTD & 0.014 & 0.008 & 0.007 & 0.006 \\
MTOP & 0.007 & 0.010 & 0.012 & 0.010 \\
DET & 0.016 & 0.003 & 0.011 & 0.007 \\
MHI & 0.001 & 0.0004 & 0.002 & 0.002 \\
\hline \hline
\end{tabular}




\section{References}

[1] F. Abe et al. (CDF Collaboration), Phys. Rev. Lett. 74, 2626 (1995); S. Abachi et al. (D0 Collaboration), Phys. Rev. Lett. 74, 2632 (1995).

[2] A. Czarnecki, J. G. Körner, J. H. Piclum, Phys. Rev. D 81, 111503 (2010). The helicity fractions used here correspond to $m_{t}=173.3 \pm 1.1 \mathrm{GeV} / c^{2}$ and $M_{W}=80.399 \pm 0.023 \mathrm{GeV} / c^{2}$, and were obtained via private communication with the authors.

[3] T. Aaltonen et al. (CDF and D0 Collaborations), Phys. Rev. D 85, 071106(R) (2012).

[4] T. Aaltonen et al., (CDF Collaboration), Phys. Rev. Lett. 105, 042002 (2010).

[5] T. Aaltonen et al., (CDF Collaboration), to be submitted.

[6] V.M. Abazov et al. (D0 Collaboration), Phys. Rev. D 83, 032009 (2011).

[7] A. Bhattia et al., Nucl. Instrum. Methods in Phys. Res. A 566, 375 (2006).

[8] V.M. Abazov et al. (D0 Collaboration), to be published in Phys. Rev. D, arXiv:1110.3771[hep-ex] (2011).

[9] Tevatron Electroweak Working Group, arXiv:1007.3178[hep-ex] (2010).

T. Sjöstrand et al., Computer Phys. Commun. 135, 238 (2001).

[10] L. Lyons, D. Gibaut, and P. Clifford, Nucl. Instrum. Methods in Phys. Res. A 270, 110 (1988).

[11] A. Valassi, Nucl. Instrum. Methods in Phys. Res. A 500, 391 (2003). 